Egyptian Journal of Aquatic Biology \& Fisheries

Zoology Department, Faculty of Science,

Ain Shams University, Cairo, Egypt.

ISSN $1110-6131$

Vol. 25(1): 423 - 441 (2021)

www.ejabf.journals.ekb.eg

\title{
Yersiniosis in Oreochromis niloticus; Prevalence, Antimicrobial Resistance and Immunological Response to Ascorbic Acid and Difloxacine
}

\author{
Salah M. Aly ${ }^{\text {* }}$, Amr Fadel ${ }^{2}$, Haidy G. Abdel-Rahman ${ }^{3}$ \\ ${ }^{1}$ Department of Pathology, College of Vet. Medicine, Suez Canal Univ., Ismailia, Egypt. \\ ${ }^{2}$ Laboratory of Fish Diseases, National Institute of Oceanography and Fisheries, Egypt. \\ ${ }^{3}$ Department of Clinical Pathology, Faculty of Veterinary medicine, Suez Canal University, Egypt
}

*Corresponding author: salahaly@ hotmail.com

ARTICLE INFO

Article History:

Received: Jan. 2, 2021

Accepted: Jan. 17, 2021

Online: Jan. 26, 2021

Keywords:

Yersinia,

Antimicrobial resistance,

Pathogenicity,

Ascorbic acid,

Interleukins,

Hematology.

\section{ABSTRACT}

Yersiniosis is a significant disease of economic importance in salmonids and non-salmonid fishes. However, few studies have investigated the pathogenesis, preventive, and treatment measures for this bacterial pathogen. Thus, the pathogenesis of Yersinia ruckeri in cultured Oreochromis niloticus in different localities was investigated throughout 2019. Besides, two experiments were conducted, the first feeding experiment based upon dietary supplementation by Ascorbic Acid and Difloxacine. In the second experiment, the supplemented fish groups were challenged by pathogenic Y. ruckeri strains, treated by Difloxacine, then the hematological, and immunological assays were analyzed. The supplemented fish groups by Ascorbic Acid and Difloxacine showed an increase in serum lysozyme, NO and SOD concentrations, phagocytic activity, and Interleukin IL-2 and IL-10 levels. The challenged fish by $Y$. ruckeri reported significant disturbances in transferases activities (ALT and AST), urea, total protein, and albumin concentrations. Moreover, RBC, $\mathrm{Hb}, \mathrm{PCV}$, nitroblue tetrazolium, lysozyme, NO and SOD concentrations, and Phagocytic activity were declined, with elevated interleukins level compared to the control. Thus, Ascorbic Acid supplementation and Difloxacine dosing could enhance the immune response, improve health status, and curtail the increased emergence of antibiotic-resistant bacteria.

\section{INTRODUCTION}

Nile Tilapia (Oreochromis niloticus) is of the most commercial fish species in Egypt and worldwide (Suresh, 2003). Despite the obstacles facing its production, but it is still considered the best native source of animal protein, which contributes to bridging the gap per capita. Disease outbreaks have been a major limitation to the dilation of aquaculture which has a significant action on the economic development in many countries (Aly, 2009; Abdel-Aziz et al., 2020; Van Doan et al., 2020; Xia et al., 2020). 
Enterobacteriaceae species; Edwardsiella tarda, E. coli, Yersinia ruckeri, and Proteus retgerri are bacterial microorganisms that cause severe economic losses among fish farms worldwide. Enteric redmouth disease (ERM) or Yersiniosis, caused by Yersinia ruckeri, Gramnegative bacterium that leads to significant economic losses in different fish species (Ross et al., 1966; Horne and Barnes, 1999). This disease was first reported in rainbow trout Oncorhynchus mykiss in the United States in 1958, then become endemic in North America (Bullock $\boldsymbol{e t}$ al., 1977; Daligault et al., 2014; Navas et al., 2014).

Other marine fish species such as turbot Scophthalmus maximus, seabass Dicentrarchus labrax, and seabream Sparus auratus have also exhibited the infection (Bullock and Cipriano, 1990). Recently, it is reported throughout North and South America, Europe, Australia, South Africa, the Middle East, and China (Tobback et al., 2007; Shaowu et al., 2013). In Egypt, $Y$. ruckeri infection has been recorded in O. niloticus (Hussein et al., 1997) and common carp and African catfish (Abd-El Latief, 2001).

The specific and non-specific immune restraints to natural or experimental infection by $Y$. ruckeri strains have been established (Anderson et al., 1979; Cossarini-Dunier, 1986; Salah et al., 2012). Following the immersion of rainbow trout by $Y$. ruckeri, high numbers of $Y$. ruckeri were attached to the gill mucus, and it was spotted within the gill capillaries, leading to septicemia and colonization of the internal organs (Tobback et al., 2009). Clinically, Yersiniosis is characterized by the development of acute and/or chronic entero-septicemia, exophthalmia, and hemorrhage on the mouth, skin, gills, and intestine (Fuhrmann et al., 1983; Rigos and Stevenson, 2001). The infection could be seen in a per-acute or acute form in young fish stages (Danley et al., 1999; Rigos and Stevenson, 2001).

Nutritional supplementation could influence the immune response of fish, play a critical role in improving health status, and subsequently provide the ability to resist diseases (Blazer, 1992; Lall, 2000; Rahimi et al., 2015). They include essential nutrients such as proteins, fatty acids, vitamins, polysaccharides, and some minerals that have vital importance to promote their immune response (Barrows et al., 2008). Vitamins $\mathrm{C}$ and $\mathrm{E}$ are among the most important nutrients which influencing the immune performance of fish (Shiau and Hsu, 2002; Puangkaew et al., 2004). Vitamin $\mathrm{C}$ is a water-soluble antioxidant and an essential microelement in biological fluids associated with biochemical reactions in cells and tissues.

Besides, antibiotics could be used in aquaculture as therapeutic and prophylactic agents (Aly, 2009; Pham et al., 2015). But, the extensive use and misuse of antibiotics as prophylactic agents share in the development and spread of antibiotic-resistant microorganisms in the environment, among human, and animal food (Cabello, 2006). Although some antimicrobials can protect against microbial infection, public health and environmental hazards must be taken into consideration.

Thus two aims were planned in this study, the first was to investigate the effect of dietary supplementation of Ascorbic acid and prophylactic antibiotics to the healthy fish and study the pathogenesis of $Y$. ruckeri in cultured $O$. niloticus in different localities. The second aim was to elucidate a treatment strategy by the antibiotic following the experimental infection of Nile 
tilapia with pathogenic $Y$. ruckeri. Consequently, the hematological, serum biochemical, and immunological parameters were assessed.

\section{MATERIAL AND METHODS}

\section{Fish for studying the pathogenesis of natural infection}

Fish sampling was performed according to the legislation of animal welfare following the guideline addressed for on humane treatment of fish (Adams et al., 2002). A total of 100 cultured $O$. niloticus with an average weight of $100 \pm 25 \mathrm{gm}$ were collected from five earthen pods farms (Farm1- Farm 5), in Sharkia Governorate to study the pathogenesis of Y. ruckeri. Then the fish were transported immediately alive in sterile polyethylene bags to the Pathology Laboratory, Faculty of Veterinary Medicine, Suez Canal University.

\section{Bacteriological examination}

The bacteriological isolates were gathered from the body surface, gills, and intestine and cultured aseptically on Tryptic Soy Broth. Following incubation at $25{ }^{\circ} \mathrm{C}$ for $48 \mathrm{~h}$, they streaked onto Heart Infusion Agar BHIA or Tryptic soy TSA media and incubated again at $25^{\circ} \mathrm{C}$ for $48 \mathrm{~h}$. Then different pure colonies were selected and sub-cultured on selective medium and Yeast Extract Glucose Agar (Michel et al., 1997) Y. ruckeri Selective Medium (Furones et al., 1993). The morphological and biochemical characterization was performed according to Waltman and Shotts, (1984), Furones et al. (1993), Elmeer et al. (1997). More confirmatory biochemical tests were performed using a commercial API 20 E system.

\section{Determination of antimicrobial resistance}

It was determined by disk diffusion on The Mueller-Hinton agar (Difco.) using six antimicrobial agents (Chloramphenicol (C $30 \mu \mathrm{g}$ ), Sulphamethoxazole \& Trimethoprim (SXT

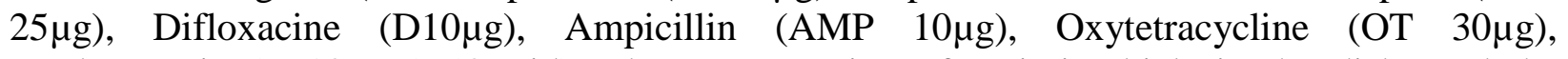
Erythromycin (E $10 \mathrm{mg}$ ) (Oxoid). The concentration of antimicrobials in the disks and the inhibition zone breakpoint values of the resistance were interpreted and the disk diffusion assays were registered according to the recommendations of the CLSI (2005 a \& b).

Experiment 1: Dietary supplementation by Ascorbic acid and Difloxacine (1-30 day)

A total of 360 healthy $O$. niloticus of $70 \pm 12 \mathrm{~g}$ body weight were assembled from a commercial farm. The fish were transported directly to the wet laboratory, then distributed in circular fiber-glassed fiber each of $1000 \mathrm{~L}$ capacity. All the requirements of the closed recirculating and filtration system, the water quality, feeding regime, and the health status of fish were managed.

Following acclimatization, the fish were divided into three triplicated groups: G1; control group fed basal diet only. G2; fed basal diet supplemented with ascorbic acid at $1000 \mathrm{mg} / \mathrm{kg}$ b.wt. (Blazer, 1992) for 30 days. G3; received basal diet till the $20^{\text {th }}$ day, then the diet was coated with Difloxacine at $5 \mathrm{mg} / \mathrm{kg}$ for 10 days (Elston et al., 1997) till the $30^{\text {th }}$ day. One month later, the blood samples were taken from the caudal blood vessels for analysis of the blood and immune parameters.

Experiment 2: Experimental infection with Y. ruckeri and Difloxacine treatment (30-40 days)

It was a complementary step for the first experiment. The control G1 was subdivided equally into G1 (control negative un-injected) and G2 (control positive injected). G2, G3, and G4 were injected intraperitoneally (I/P) with $0.5 \mathrm{ml}$ of a bacterial suspension of $Y$. ruckeri at $1.5 \mathrm{x}$ $10^{8} \mathrm{CFU} /$ fish. Two days later, the injected groups were treated with Difloxacine $10 \mathrm{mg} / \mathrm{L}$, one 
hour bath, for 10 successive days. Along the treatment course, the clinical signs and mortalities were registered daily. Following the treatment period, the blood samples were taken and the hematological, and immunological parameters were analyzed.

\section{Clinical signs}

Either clinical internal or external abnormalities, besides, physical and behavioral signs were reported according to Danley et al. (1999).

\section{Blood sampling}

Blood samples were drawn from the caudal vessels and directly placed in a tube containing potassium salt of EDTA and used as whole blood for hematological examination. The remaining amount of blood was placed in a plain clean tube and centrifuged at $3000 \mathrm{rpm}$ for 5 minutes then the serum was separated for the biochemical and immunological assays.

\section{Hematological examination}

The erythrocytic and total leukocytic counts and the blood films were set for the differential leukocytic count with Giemsa stain according to Blaxhall and Daisley, (1973). Hemoglobin concentration $(\mathrm{Hb})$ was determined by the cyanomethemoglobin method (Dorafshan et al., 2008).

\section{Serum biochemical and immunological parameters}

Serum levels of total proteins, albumin, glucose, urea, creatinine, uric acid, and transferases (ALT \& AST) activities were analyzed according to Koller, (1984), Bartholomew and Delaney, (1964); Young (2001), Tietz (1995); Bartels and Bohmer, (1972), and Reitman and Frankel, (1957) respectively. Also, lysozyme concentration was assessed by turbidimetric assay, (Ellis, 1990), Nitroblue tetrazolium (NBT) (Glasser and Fiederlein, 1990), Nitric oxide (Green, et al., 1982). IL-2 and IL-10 were measured using ELISA kits manufactured by Biosource Inc., San Diego, California, USA.

\section{$\underline{\text { Statistical analysis }}$}

Statistical analysis was performed on mean values using one-way analysis of variance (ANOVA) with Duncan Multiple Range Test was done to determine the differences among fish groups at the significance level of $P<0.05$ (Duncan, 1955) by using SPSS for Windows, version 15 , USA. The standard errors were estimated. Data of antimicrobial resistance was analyzed with a chi-squared test according to the SAS package (SAS, 2005).

\section{RESULTS}

\section{Bacteriological identification}

The recovered Y. ruckeri strains develop raised round, shiny, entire, and off-white colonies of 2-3 mm diameter. Most of the biochemical tests showed homogenous patterns except for Voges-Proskauer, gelatin, and sorbitol (Table 1).

\section{The prevalence of Yersinia ruckeri infection}

As shown in Table 2, the isolated $Y$. ruckeri strains from fish body surface and gills were reported in the five farms with a total prevalence of 7\%. Except, Farm 2, the intestinal bacterial isolates recorded a 5.63\% prevalence rate (Table 3). 
Table (1): Bacteriological identification of $Y$. ruckeri isolates.

\begin{tabular}{|c|c|c|c|c|c|}
\hline Test & Farm1 & Farm 2 & Farm 3 & Farm 4 & Farm 5 \\
\hline OX & - & - & - & - & - \\
\hline CAT & + & + & + & + & + \\
\hline MOT & + & + & + & + & + \\
\hline ONPG & + & + & + & + & + \\
\hline ADH & - & - & - & - & - \\
\hline LDC & + & + & + & + & + \\
\hline ODC & + & + & + & + & + \\
\hline CIT & - & - & - & - & - \\
\hline H2S & - & - & - & - & - \\
\hline URE & - & - & - & - & - \\
\hline TDA & - & - & - & - & - \\
\hline IND & - & - & - & - & - \\
\hline GLU & + & + & + & + & + \\
\hline MAN & + & + & + & + & + \\
\hline INO & - & - & - & - & - \\
\hline RHA & - & - & - & - & - \\
\hline SUC & - & - & - & - & - \\
\hline MEL & - & - & - & - & - \\
\hline AMY & - & - & - & - & - \\
\hline ARA & - & - & - & - & - \\
\hline SOR & - & + & + & - & - \\
\hline $\mathbf{G}$ & - & + & - & - & - \\
\hline $\mathbf{V P}$ & + & + & + & - & + \\
\hline NO2 & + & + & + & + & + \\
\hline N2 & + & + & + & + & + \\
\hline $\mathbf{O}$ & + & + & + & + & + \\
\hline $\mathbf{F}$ & + & + & + & + & + \\
\hline
\end{tabular}

OX: oxidase, CAT: catalase, $\beta \mathrm{H}$ : b haemolysis, MOT: motility, ONPG: b-galactosidase, ADH: arginine dihyrolase, LDC: lysine decarboxylase, ODC: ornithine decarboxylase, CIT: citrate, H2S: production of hydrogen sulphide, URE: urease,TDA: tryptophanedeaminase, IND: indole, VP: Voges-Proskauer, G: gelatin, GLU: glucose, MAN: mannitol, INO: inositol, SOR:sorbitol, RHA: rhamnose, SUC: sucrose, MEL: melibiose, AMY: amygdalin, ARAB: arabinose, NO2: nitrate reduction, N2: reduction to nitrite gas.

\section{Antimicrobial resistance}

The antimicrobial susceptibility of $Y$. ruckeri strains showed a higher resistance pattern for all antibiotics except for Difloxacine. The multi-resistant patterns of Y.ruckeri were shown in Table 4. 


\section{Clinical signs}

The infected fish exhibited behavioral changes including surface swimming, lethargy, and decreased appetite. The common signs were bilateral exophthalmia, inflammation, erosion, darkened skin, and hemorrhages around the mouth (red mouth) also appeared. Low sustained mortalities were observed which increased over time. Internally, enlarged liver and kidney, and intestine filled with a yellowish fluid of fetid, besides, petechial hemorrhage in the liver, spleen, intestine could be noticed.

Table (2): Identification and source of selected bacterial isolates from the body surface and gills of farmed Nile tilapia $(\mathrm{n}=100$ out of 5 farms).

\begin{tabular}{|lcccccc|}
\hline Type of isolates & TF1 & TF2 & TF3 & TF4 & TF5 & Total \\
\hline Yersinia ruckeri & 1 & 1 & 2 & 2 & 1 & 7 \\
\hline
\end{tabular}

Table (3): Identification of the intestinal bacterial isolates from examined Nile tilapia $(\mathrm{n}=71)$.

\begin{tabular}{|ccccccc|}
\hline Type of isolates & TF1 & TF2 & TF3 & TF4 & TF5 & Total \\
\hline Yersinia ruckeri & 1 & - & 1 & 1 & 1 & 4 \\
\hline
\end{tabular}

Table (4): Antimicrobial resistance of $Y$. ruckeri strains ( $\mathrm{n}=56$ isolates).

\begin{tabular}{|ccccccc|}
\hline Bacterial strains & \multicolumn{7}{c|}{ Antimicrobial resistance (\%) } \\
\hline Farm & C & SXT & D & AMP & OT & E \\
\hline F1 & 20 & 70 & 0 & 70 & 100 & 80 \\
F2 & 60 & 40 & 20 & 70 & 100 & 90 \\
F3 & 70 & 80 & 30 & 80 & 100 & 100 \\
F4 & 40 & 60 & 30 & 60 & 80 & 90 \\
F5 & 50 & 30 & 10 & 10 & 60 & 50 \\
Chi square value & $11.2^{* *}$ & $7.5^{*}$ & $6.7^{*}$ & $0.68^{\mathrm{ns}}$ & $0^{\mathrm{ns}}$ & $4.44^{\mathrm{ns}}$ \\
\hline
\end{tabular}

C: Chloramphenicol, SXT: Sulphamethoxazole/Trimethoprim, D: Difloxacine, Am: Amplicllin, OT: Oxytetracycline, E: Erythromycin. *: P value $<0.05$ (significant), **: P value $<0.01$ (highly significant), ns: $\mathrm{P}$ value $>0.05$ (non-significant) 
Table (5): The eight most common antimicrobial resistance profiles encountered in 81 multiresistant $Y$. ruckeri strains from gills of Nile tilapia.

\begin{tabular}{|lc|}
\hline \multicolumn{1}{|c|}{ Antibiotic resistance profile } & No. of Y. ruckeri strains \\
\hline OT, SXT, AMP, E & 5 \\
OT, SXT, C & 3 \\
\hline
\end{tabular}

\section{Experiment 1: Dietary supplementation by Ascorbic acid and Difloxacine}

\subsection{Hematological parameters}

As shown in Table 6, $\mathrm{Hb}$ concentration was significantly $(P<0.05)$ higher in the Ascorbic Acid-supplemented group than Difloxacine-treated group. RBCs and PCV were non significantly changed in all groups. Additionally, the supplemented groups revealed significant $(P<0.05)$ leukocytosis, neutrophilia, and monocytosis in correspondence to the control.

Table (6): Some hematological parameters (mean values \pm SE) at $30^{\text {th }}$ day following dietary supplementation of Nile tilapia with Ascorbic acid and Difloxacine.

\begin{tabular}{|c|c|c|c|}
\hline \multirow[b]{2}{*}{ Parameters } & \multicolumn{3}{|c|}{ Experimental groups } \\
\hline & $\begin{array}{c}\text { G1 } \\
\text { Control }\end{array}$ & $\begin{array}{c}\text { G2 } \\
\text { Ascorbic Acid }\end{array}$ & $\begin{array}{c}\text { G3 } \\
\text { Difloxacine }\end{array}$ \\
\hline $\operatorname{RBCs}(10 \% / \mu \mathrm{l})$ & $2.28 \pm 0.04^{\mathrm{a}}$ & $2.37 \pm 0.05^{\mathrm{a}}$ & $2.40 \pm 0.11^{\mathrm{a}}$ \\
\hline $\mathrm{Hb}(\mathrm{g} / \mathrm{dl})$ & $5.84 \pm 0.28^{b}$ & $6.88 \pm 0.16^{\mathrm{a}}$ & $5.81 \pm 0.17^{\mathrm{b}}$ \\
\hline $\operatorname{PCV}(\%)$ & $21.67 \pm 0.88^{\mathrm{a}}$ & $21.00 \pm 0.58^{\mathrm{a}}$ & $20.00 \pm 0.58^{\mathrm{a}}$ \\
\hline $\operatorname{MCV}(\mathbf{f l})$ & $95.30 \pm 4.92^{\mathrm{a}}$ & $88.52 \pm 4.04^{\mathrm{a}}$ & $83.93 \pm 6.40^{\mathrm{a}}$ \\
\hline MCH(pg) & $25.67 \pm 1.14^{\mathrm{a}}$ & $28.96 \pm 0.37^{\mathrm{a}}$ & $24.37 \pm 1.87^{\mathrm{a}}$ \\
\hline MCHC(\%) & $27.17 \pm 2.41^{\mathrm{b}}$ & $32.82 \pm 1.15^{\mathrm{a}}$ & $29.03 \pm 0.12^{\mathrm{ab}}$ \\
\hline $\operatorname{TLC}\left(10^{3} / \mu \mathrm{I}\right)$ & $36.21 \pm 1.23^{\mathrm{b}}$ & $39.68 \pm 0.49^{\mathrm{a}}$ & $38.67 \pm 0.29^{\mathrm{ab}}$ \\
\hline Neutrophils $\left(10^{3} / \mu \mathrm{l}\right)$ & $15.42 \pm 1.76^{\mathrm{b}}$ & $20.66 \pm 0.98^{\mathrm{a}}$ & $21.07 \pm 0.15^{\mathrm{a}}$ \\
\hline Lymphocytes $\left(10^{3} / \mu \mathrm{l}\right)$ & $19.27 \pm 0.51^{\mathrm{a}}$ & $17.28 \pm 1.01^{\mathrm{ab}}$ & $15.84 \pm 0.38^{\mathrm{b}}$ \\
\hline $\operatorname{Monocytes}\left(10^{3} / \mu \mathrm{l}\right)$ & $1.18 \pm 0.10^{\mathrm{b}}$ & $1.47 \pm 0.07^{\mathrm{a}}$ & $1.55 \pm 0.08^{\mathrm{a}}$ \\
\hline Eosinophils $\left(10^{3} / \mu \mathrm{l}\right)$ & $0.34 \pm 0.05^{\mathrm{a}}$ & $0.26 \pm 0.04^{\mathrm{a}}$ & $0.21 \pm 0.02^{\mathrm{a}}$ \\
\hline
\end{tabular}

RBCs: Red Blood Cells, Hb; Hemoglobin, PCV: Packed Cell Volume, MCV: Mean Corpuscular Volume, MCH: Mean Corpuscular Hemoglobin, MCHC: Mean Corpuscular Hemoglobin Concentration, TLC: Total Leucocytic Count.

\subsection{Serum biochemical parameters}

Similarly, the Ascorbic Acid and Difloxacine-supplemented groups showed nonsignificant changes in all estimated serum biochemical analytes compared to the control (Table 7). 
Table (7): Some serum biochemical analytes (mean values \pm SE) at $30^{\text {th }}$ day following dietary supplementation of Nile tilapia with Ascorbic acid and Difloxacine.

\begin{tabular}{|lccc|}
\hline & \multicolumn{3}{c|}{ Experimental groups } \\
\cline { 2 - 4 } Parameters & G1 & G2 & G3 \\
& Control & Ascorbic Acid & Difloxacine \\
\hline AST (U/L) & $7.52 \pm 0.99^{\mathrm{a}}$ & $8.50 \pm 0.45^{\mathrm{a}}$ & $9.86 \pm 0.74^{\mathrm{a}}$ \\
ALT(U/L) & $9.03 \pm 1.15^{\mathrm{a}}$ & $9.12 \pm 0.32^{\mathrm{a}}$ & $10.75 \pm 0.70^{\mathrm{a}}$ \\
TP (g/dl) & $6.00 \pm 0.09^{\mathrm{a}}$ & $6.11 \pm 0.03^{\mathrm{a}}$ & $5.39 \pm 0.44^{\mathrm{a}}$ \\
Albumin (g/dl) & $4.11 \pm 0.06^{\mathrm{a}}$ & $3.38 \pm 0.16^{\mathrm{a}}$ & $3.96 \pm 0.08^{\mathrm{a}}$ \\
Glucose(mg/dl) & $65.52 \pm 2.58^{\mathrm{a}}$ & $67.53 \pm 1.86^{\mathrm{a}}$ & $64.61 \pm 3.03^{\mathrm{a}}$ \\
Uric acid (mg/dl) & $0.95 \pm 0.06^{\mathrm{a}}$ & $0.94 \pm 0.03^{\mathrm{a}}$ & $0.94 \pm 0.04^{\mathrm{a}}$ \\
Urea (mg/dl) & $16.72 \pm 0.59^{\mathrm{a}}$ & $18.18 \pm 0.95^{\mathrm{a}}$ & $18.58 \pm 1.18^{\mathrm{a}}$ \\
Creatinine & $43.92 \pm 2.80^{\mathrm{a}}$ & $41.71 \pm 2.18^{\mathrm{a}}$ & $43.77 \pm 2.27^{\mathrm{a}}$ \\
(mg/dl) & & & \\
\hline
\end{tabular}

AST: Aspartate aminotransferase, ALT: Alanine aminotransferase, TP: Total Protein.

\subsection{The immunological assays}

Significant $(P<0.05)$ elevations were showed in Lysozyme, NO, Phagocytic activity \%, IL2, IL10, and SOD concentration in the Ascorbic Acid and Difloxacine- supplemented groups compared to the control. While NBT was non significantly increased in the supplemented groups (Table 8).

Table (8): Some immunological assays (mean values \pm SE) at $30^{\text {th }}$ following dietary supplementation of Nile tilapia with Ascorbic acid and Difloxacine.

\begin{tabular}{|lccc|}
\hline \multirow{2}{*}{ Parameters } & \multicolumn{3}{c|}{ Experimental groups } \\
\cline { 2 - 4 } & $\begin{array}{c}\text { G1 } \\
\text { Control }\end{array}$ & $\begin{array}{c}\text { G2 } \\
\text { Ascorbic Acid }\end{array}$ & $\begin{array}{c}\text { G3 } \\
\text { Difloxacine }\end{array}$ \\
\hline NBT(OD) & $0.96 \pm 0.05^{\mathrm{a}}$ & $0.99 \pm 0.03^{\mathrm{a}}$ & $0.97 \pm 0.03^{\mathrm{a}}$ \\
Lysozyme(U/L) & $82.12 \pm 3.62^{\mathrm{b}}$ & $109.75 \pm 6.05^{\mathrm{a}}$ & $103.77 \pm 4.63^{\mathrm{a}}$ \\
NO( $\boldsymbol{\mu g} / \mathbf{d l})$ & $25.28 \pm 1.58^{\mathrm{b}}$ & $41.67 \pm 1.31^{\mathrm{a}}$ & $37.13 \pm 2.36^{\mathrm{a}}$ \\
SOD(U/ml) & $249.08 \pm 2.95^{\mathrm{b}}$ & $270.41 \pm 4.29^{\mathrm{a}}$ & $261.05 \pm 3.55^{\mathrm{a}}$ \\
Phagocytic(\%) & $52.38 \pm 1.87^{\mathrm{b}}$ & $83.63 \pm 2.18^{\mathrm{a}}$ & $75.93 \pm 3.00^{\mathrm{a}}$ \\
IL-2(Pg/ml) & $4.19 \pm 0.22^{\mathrm{c}}$ & $5.18 \pm 0.24^{\mathrm{b}}$ & $7.72 \pm 0.23^{\mathrm{a}}$ \\
IL-10(Pg/ml) & $3.11 \pm 0.13^{\mathrm{c}}$ & $4.04 \pm 0.22^{\mathrm{b}}$ & $6.38 \pm 0.21^{\mathrm{a}}$ \\
Mortality rate & $3.33 \pm 1.67^{\mathrm{a}}$ & $1.67 \pm 1.67^{\mathrm{a}}$ & $3.33 \pm 3.33^{\mathrm{a}}$ \\
\hline
\end{tabular}

NBT: Nitroblue tetrazolium, NO: Nitric Oxide, SOD: Superoxide Dismutase, IL: Interleukin. 


\section{Experiment 2: Experimental infection with Yersinia ruckeri and Difloxacine treatment}

\subsection{Hematological parameters}

On the $40^{\text {th }}$ day, the Yersinia-infected fish revealed a significant decrease in RBCs, Hb content, and PCV compared to the control and treated fish (Table 9).

\subsection{Serum biochemical parameters}

A significant $(P<0.05)$ increase in AST activity in Yersinia-infected fish compared to the control. Meanwhile, the treated groups showed a numerical decline in AST activity compared to the infected group. Furthermore, the infected group manifested a significant $(P<0.05)$ increase in ALT activity and urea level in comparison to the control and treated groups. Significant $(P<0.05)$ hypoproteinemia and hypoalbuminemia were detected in the infected group in comparison to the control and treated groups. As for creatinine, uric acid, and glucose levels, there were non-significant variations between all groups, except for a numerical increase in creatinine level in Yersinia infected group (Table 10).

Table (9): Some hematological parameters (mean values \pm SE) at $40^{\text {th }}$ day in the healthy control and $Y$. ruckeri-infected fish supplemented with Ascorbic acid and Difloxacine.

\begin{tabular}{|c|c|c|c|c|}
\hline \multirow{2}{*}{ Parameters } & \multicolumn{4}{|c|}{ Experimental groups } \\
\hline & G1 & G2 & G3 & G4 \\
\hline $\operatorname{RBCs}(10 \% / \mu \mathrm{l})$ & $2.52 \pm 0.18^{\mathrm{a}}$ & $1.21 \pm 0.06^{b}$ & $2.43 \pm 0.08^{\mathrm{a}}$ & $2.41 \pm 0.06^{\mathrm{a}}$ \\
\hline $\mathrm{Hb}(\mathrm{g} / \mathrm{dl})$ & $6.51 \pm 0.16^{\mathrm{a}}$ & $5.18 \pm 0.09^{b}$ & $7.26 \pm 0.77^{\mathrm{a}}$ & $6.82 \pm 0.06^{\mathrm{a}}$ \\
\hline $\operatorname{PCV}(\%)$ & $21.33 \pm 0.67^{\mathrm{a}}$ & $16.00 \pm 0.58^{\mathrm{b}}$ & $21.67 \pm 0.88^{\mathrm{a}}$ & $20.67 \pm 1.45^{\mathrm{a}}$ \\
\hline $\operatorname{MCV}(\mathbf{f l})$ & $84.75 \pm 2.25^{\mathrm{b}}$ & $133.08 \pm 6.13^{\mathrm{a}}$ & $89.17 \pm 3.97^{b}$ & $86.02 \pm 7.49^{b}$ \\
\hline $\operatorname{MCH}(p g)$ & $25.89 \pm 0.78^{\mathrm{b}}$ & $43.06 \pm 1.50^{\mathrm{a}}$ & $29.71 \pm 2.13^{b}$ & $28.31 \pm 0.46^{\mathrm{b}}$ \\
\hline МCHC(\%) & $30.64 \pm 1.73^{\mathrm{a}}$ & $32.41 \pm 0.88^{\mathrm{a}}$ & $33.50 \pm 3.05^{\mathrm{a}}$ & $33.35 \pm 2.57^{\mathrm{a}}$ \\
\hline $\operatorname{TLC}\left(10^{3} / \mu \mathrm{l}\right)$ & $37.24 \pm 0.55^{\mathrm{ab}}$ & $30.87 \pm 2.14^{\mathrm{b}}$ & $35.15 \pm 2.87^{\mathrm{ab}}$ & $39.97 \pm 2.70^{\mathrm{a}}$ \\
\hline Neutrophils $\left(10^{3} / \mu \mathrm{l}\right)$ & $14.78 \pm 0.24^{\mathrm{ab}}$ & $9.40 \pm 0.45^{\mathrm{b}}$ & $19.62 \pm 2.04^{\mathrm{a}}$ & $19.49 \pm 2.98^{\mathrm{a}}$ \\
\hline Lymphocytes $\left(10^{3} / \mu \mathrm{I}\right)$ & $21.00 \pm 0.27^{\mathrm{a}}$ & $19.96 \pm 1.62^{\mathrm{a}}$ & $13.92 \pm 0.95^{\mathrm{b}}$ & $18.60 \pm 0.36^{\mathrm{a}}$ \\
\hline Monocytes $\left(10^{3} / \mu \mathrm{l}\right)$ & $1.05 \pm 0.08^{\mathrm{b}}$ & $1.15 \pm 0.08^{\mathrm{b}}$ & $1.32 \pm 0.08^{\mathrm{ab}}$ & $1.57 \pm 0.19^{\mathrm{a}}$ \\
\hline Eosinophils $\left(10^{3} / \mu \mathrm{l}\right)$ & $0.40 \pm 0.04^{\mathrm{a}}$ & $0.36 \pm 0.01^{\mathrm{ab}}$ & $0.28 \pm 0.02^{\mathrm{b}}$ & $0.30 \pm 0.01^{\mathrm{b}}$ \\
\hline
\end{tabular}

RBCs: Red Blood Cells, Hb: Hemoglobin, PCV: Packed Cell Volume, MCV: Mean Corpuscular Volume, MCH: Mean Corpuscular Hemoglobin, MCHC: Mean Corpuscular Hemoglobin Concentration, TLC: Total Leucocytic Count. G1: Control un- challenged group, G2: Control challenged group, G3: challenged Ascorbic Acid-treated group, and G4: challenged Difloxacine treated group. 
Table (10): Some serum biochemical analytes (mean values \pm SE) at $40^{\text {th }}$ day in healthy control and Y. ruckeri-infected fish supplemented with Ascorbic acid and Difloxacine.

\begin{tabular}{|lcccc|}
\hline \multirow{2}{*}{ Parameters } & \multicolumn{4}{c|}{ Experimental groups } \\
\cline { 2 - 5 } & G1 & G2 & G3 & G4 \\
\hline AST (U/L) & $9.68 \pm 0.62^{\mathrm{b}}$ & $15.57 \pm 1.91^{\mathrm{a}}$ & $10.90 \pm 1.23^{\mathrm{ab}}$ & $13.53 \pm 2.31^{\mathrm{ab}}$ \\
ALT(U/L) & $11.98 \pm 1.17^{\mathrm{b}}$ & $22.38 \pm 1.55^{\mathrm{a}}$ & $11.59 \pm 0.75^{\mathrm{b}}$ & $11.18 \pm 1.61^{\mathrm{b}}$ \\
TP (g/dl) & $5.83 \pm 0.17^{\mathrm{a}}$ & $3.50 \pm 0.32^{\mathrm{b}}$ & $5.22 \pm 0.80^{\mathrm{a}}$ & $5.32 \pm 0.63^{\mathrm{a}}$ \\
Albumin (g/dl) & $5.33 \pm 0.19^{\mathrm{a}}$ & $2.18 \pm 0.22^{\mathrm{b}}$ & $4.87 \pm 0.11^{\mathrm{a}}$ & $4.98 \pm 0.51^{\mathrm{a}}$ \\
Glucose & $50.82 \pm 2.67^{\mathrm{a}}$ & $64.87 \pm 3.35^{\mathrm{a}}$ & $55.06 \pm 6.05^{\mathrm{a}}$ & $52.51 \pm 4.49^{\mathrm{a}}$ \\
(mg/dl) & & & & \\
Uric acid & $1.26 \pm 0.19^{\mathrm{ab}}$ & $1.41 \pm 0.27^{\mathrm{a}}$ & $0.79 \pm 0.05^{\mathrm{b}}$ & $0.94 \pm 0.05^{\mathrm{ab}}$ \\
(mg/dl) & & & & \\
Urea (mg/dl) & $15.09 \pm 0.28^{\mathrm{b}}$ & $22.80 \pm 1.77^{\mathrm{a}}$ & $17.52 \pm 0.84^{\mathrm{b}}$ & $18.19 \pm 0.50^{\mathrm{b}}$ \\
Creatinine & $37.47 \pm 0.26^{\mathrm{a}}$ & $48.16 \pm 4.80^{\mathrm{a}}$ & $39.04 \pm 1.67^{\mathrm{a}}$ & $45.38 \pm 3.90^{\mathrm{a}}$ \\
(mg/dl) & & & & \\
\hline AST:Aspartat & & & & \\
\hline
\end{tabular}

AST: Aspartate aminotransferase, ALT: Alanine aminotransferase, TP: Total Protein. G1: Control unchallenged group. G2: Control challenged group, G3: challenged Ascorbic Acid-treated group, and G4: challenged Difloxacine treated group.

Table (11): Some immunological assays (mean values $\pm \mathrm{SE}$ ) at $40^{\text {th }}$ day in healthy control and $Y$. ruckeri-infected fish supplemented with Ascorbic acid and Difloxacine.

\begin{tabular}{|lcccc|}
\hline \multirow{2}{*}{ Parameters } & \multicolumn{4}{c|}{ Experimental groups } \\
\cline { 2 - 5 } & G1 & G2 & G3 & G4 \\
\hline NBT(OD) & $0.92 \pm 0.02^{\mathrm{a}}$ & $0.78 \pm 0.02^{\mathrm{b}}$ & $0.97 \pm 0.03^{\mathrm{a}}$ & $0.90 \pm 0.01^{\mathrm{a}}$ \\
Lysozyme(U/L) & $82.09 \pm 1.66^{\mathrm{a}}$ & $10.28 \pm 0.65^{\mathrm{c}}$ & $53.14 \pm 2.11^{\mathrm{b}}$ & $52.21 \pm 1.60^{\mathrm{b}}$ \\
NO( $\boldsymbol{\mu g} / \mathbf{d l})$ & $21.74 \pm 1.36^{\mathrm{a}}$ & $2.05 \pm 0.38^{\mathrm{c}}$ & $15.68 \pm 0.90^{\mathrm{b}}$ & $13.02 \pm 1.48^{\mathrm{b}}$ \\
SOD(U/ml) & $246.43 \pm 3.81^{\mathrm{b}}$ & $218.52 \pm 7.46^{\mathrm{c}}$ & $266.04 \pm 3.44^{\mathrm{a}}$ & $255.88 \pm 3.84^{\mathrm{ab}}$ \\
Phagocytic(\%) & $41.85 \pm 1.40^{\mathrm{b}}$ & $21.49 \pm 1.29^{\mathrm{c}}$ & $72.60 \pm 1.85^{\mathrm{a}}$ & $73.96 \pm 2.82^{\mathrm{a}}$ \\
IL-2(Pg/ml) & $5.00 \pm 0.45^{\mathrm{b}}$ & $7.14 \pm 0.21^{\mathrm{a}}$ & $4.82 \pm 0.20^{\mathrm{b}}$ & $3.87 \pm 0.19^{\mathrm{c}}$ \\
IL-10(Pg/ml) & $4.53 \pm 0.37^{\mathrm{b}}$ & $5.79 \pm 0.15^{\mathrm{a}}$ & $3.58 \pm 0.30^{\mathrm{c}}$ & $3.25 \pm 0.14^{\mathrm{c}}$ \\
Mortality rate & $1.67 \pm 1.67^{\mathrm{c}}$ & $61.67 \pm 6.01^{\mathrm{a}}$ & $36.67 \pm 3.33^{\mathrm{b}}$ & $38.33 \pm 3.33^{\mathrm{b}}$ \\
\hline
\end{tabular}

NBT; Nitroblue tetrazolium, NO; Nitric Oxide, SOD; Superoxide Dismutase, IL; Interleukin. G1: Control un-challenged group, G2: Control challenged group, G3: challenged Ascorbic Acid-treated group, and G4: challenged Difloxacine treated group.

\subsection{The immunological assays}

In the Yersinia-infected group, NBT, Phagocytic \%, lysozyme activity, NO level and SOD activity were significantly $(P<0.05)$ decreased and IL2 and IL10 were significantly $(P<0.05)$ increased in comparison to the control and treated groups (Table 11). 


\section{DISCUSSION}

Throughout this study, some of the epidemiological characteristics of Yersinia ruckeri infection in Nile tilapia Oreochromis niloticus such as occurrence, outbreaks, and prevalence in organs were known. The behavior of the disease was also defined experimentally, in terms of its clinical signs, postmortem, and mortality rate. More importantly, is the evaluation of the hematological, biochemical, and immunological activities, during the infection, and posttreatment.

Yersinia, a genus of the Enterobacteriaceae family, comprises several species, which cause diseases in humans, animals, and fish. The infected fish exhibited some behavioral, clinical, and postmortem changes which were most similar to the previously described by Danley et al. (1999).

The bacteriological identification of bacterial isolates manifested Gram-negative bacilli, oxidase-negative, catalase-positive glucose and mannitol fermenter, nitrate-reductive, secrete lysine, b-galactosidase, and ornithine decarboxylases but neither indole nor hydrogen sulfide. Similar biochemical patterns were identified by Furones et al. (1993) and EImeer et al. (1997). Also, the identified strains from different farms were largely homogenous, with some biochemical variation that might be related to the genetic diversity within $Y$. ruckeri strains (Bastardo et al., 2011).

The spatial occurrence of $Y$. ruckeri infection showed significant prevalences in the different farms. This suggests the epizootic of the infection and the high adaptation of exploding to be widely distributed, moreover, this assures the evolutionary relationships among diverse bacterial species. Thus $Y$. ruckeri has a wide range of locality and host that could explain their ability to persist longer in the aquatic environment (Romalde et al., 1994), and transmission through carriers to the healthy fish at an optimum temperature under any stressors (Hunter $\boldsymbol{e t}$ al., 1980).

By screening the susceptibility of $Y$. ruckeri isolates, they were showed resistance patterns to OT, SXT, AMP, and E. Similar resistance was interpreted by Rodgers, (2001) in oxolinic acid, oxytetracycline, and potentiated sulphonamide. He has attributed it to $Y$. ruckeri $\beta$ lactamase gene which is not likely to be expressed at high levels (Stock et al., 2002; Mammeri et al., 2006). Oppositly, Klein et al. (1996) did not found resistance patterns among $Y$. ruckeri isolates, except for a lower sensitivity to OXA. Thus, the pathogenic strains evolve different antimicrobial resistance due to misuse of antimicrobial agents.

Many factors could be contributed to pathogenicity and the appearance of external, internal signs, and mortalities. They could be related to the generalized septicemia and the widespread of the pathogen in all organs, including the brain (Ohtani et al., 2014). Méndez and Guijarro, (2013) revealed that some promotors such as $y h l B A, \operatorname{cds} A B, y c t C B A$, and $y r p l$ were expressed and led to hemolysin production, cysteine and citrate uptake, and the encoding of serralysin metalloprotease, respectively. Other factors have been linked to the pathogenicity such as a serralysin metalloprotease (metalloendopeptidase), termed Yrp1, that hydrolyses actin, fibrinogen, gelatin, laminin, and myson (but not type II and type IV collagen) (Fernandez-Diaz et al., 2003). 
The hematological parameters of yersinia-infected fish recorded macrocytic normochromic anemia. Our result came in agreement with Altun and Diler, (1999) who reported macrocytic normochromic anemia in rainbow trout experimentally infected with $Y$. ruckeri on day 13 and 15. Macrocytic anemia takes place due to the failure of DNA division of the erythrocytic cell (Hoffman et al., 2009).

Vitamin $\mathrm{C}$ is a necessary immunostimulant facing bacteria and virus-infected fish and is responsible for the proliferation and phagocytosis of the immune cells (Ergönül et al., 2012). Tewary and Patra, (2008) have concluded that high doses of vitamin C (500 to $2000 \mathrm{mg} / \mathrm{kg}$ ) in rainbow trout stimulated hematopoiesis, raised the immune response, and repaired tissue damage against most viral and bacterial pathogens including $Y$. ruckeri.

At the same time, an increase mainly in serum activities of ALT, AST, and level of urea was reported along with hypoproteinemia and hypoalbuminemia in the infected non-treated group. These marked changes could be attributed to the marked degenerative changes that have been noticed in the infected tissues of the liver and kidneys (Salah $\boldsymbol{e t}$ al., 2012). It has been previously discussed that the increased serum liver transaminases (ALT and AST) may elucidate hepatocellular injury and inflammatory reaction leading to the emission of these enzymes into the blood (Fuchs et al., 1986). Furthermore, serum ALT and AST activities are counted as sensitive signals to estimate hepatic and myocardial deterioration (Raa, 1994). At the same pace, the obtained results of serum hypoproteinemia and hypoalbuminemia confirmed the occurrence of liver disorder due to stress conditions and bacterial infection (Ellis, 1981).

Moreover, some immunological assays such as NBT, Phagocytic \%, NO, Lysozyme, and SOD activities showed declination in the infected group. The potency of the phagocytic cells neutrophils, monocytes, and macrophages is associated with activation of the innate immune response (Ayub et al., 2003). Neutrophils are an essential partner of the host defense and valuable for the assessment of the fish's health status. The macrophages are considered a focal part of the cellular non-specific defense. An inflammatory reaction has been shown in the body cavity in the $Y$. ruckeri infected rainbow trout (Afonso et al., 1998), due to the flow of large numbers of neutrophils, caught from the blood and hemopoietic tissue. Neutrophils actively share in the nonspecific immunity of the fish by producing reactive oxygen species or radicals for killing bacteria, then Nitro-blue tetrazolium (NBT) reacts with these oxidative radicals producing a dark blue stain (Anderson et al., 1992; Barman et al., 2013). Therefore, the phagocytic activity of neutrophils can be positively correlated to the NBT activity.

Another defense element, Superoxide dismutase enzyme (SOD) which is detected in erythrocytes and the liver and prevent oxidative fatigue of the cells. High SOD activity protects the cells against oxidative stress caused by bacterial infections. Where, it is concerned with defensive mechanisms against oxidative stress as that provoked by intracellular pathogens like $Y$. ruckeri (Guijarro et al., 2018).

Nitric oxide (NO) is bactericidal reactive oxygen that is produced by phagocytes (macrophages, monocytes, and neutrophils) following microbial invasion (Campos-Perez et al., 2000). NO was found to cause DNA damage of the pathogen (Juedes and Wogan, 1996) and adjust the immune response toward the pathogen. 
Lysozyme is an index of the innate immunity of the fish and one of the defense mechanisms of the body toward microbial invasion, due to its anti bactericidal impact (Jollès and Jollès, 1984) against different bacterial species (Saurabh and Sahoo, 2008). It can break the chemical bond between the $\mathrm{N}$-acetylglucosamine and $\mathrm{N}$-acetylmuramic acid of the peptidoglycan in bacterial cell walls, and mainly secreted by macrophages and neutrophils (Nathan, 1987). Jaafar et al. (2013) stated that experimentally infected fish with $Y$. ruckeri exhibited a reduction in the lysozyme activity after 45 days and this reduction may be attributed to exhaustion of the plasma lysozyme in fish blood facing the bacterial invasion. In a challenge test with vibriosis, a negative correlation between serum lysozyme activity and survival has been observed in Atlantic salmon (Roed et al., 1993).

On the other hand, ascorbic acid and antibiotic supplementation to the infected groups resulted in enhanced activity of lysozymes in comparison to the infected non-treated group. Increased lysozyme activity can be attributed to the dietary supplements for fish, through the synergistic effect on the immune system due to the increase in the phagocyte number elevates lysozyme synthesis by cells (Kumari and Sahoo, 2006).

Furthermore, IL2 and IL10 showed an elevation in their levels in the infected non-treated group. The inflammatory response in $Y$. ruckeri infected rainbow trout reported raised levels of inflammatory cytokines such as interleukin (IL)- 1 family members, IL-6, IL-8, IL-10, and IFN- $\gamma$ (Raida and Buchmann, 2008; Wang et al., 2009; Kumar et al., 2015). Interleukin IL-2 is an immunomodulatory element that helps proliferation, differentiation, and activation of $\mathrm{T}$ cells (Sogo et al., 2009). It is synthesized mainly by Th1 cells and is known as the T-cell growth factor (TCGF) (Smith, 1988). Also, the expression of Interleukin IL-10 can be increased by a bacterial infection, LPS stimulation, and immunostimulants bath supplementation (Zhang et al., 2009). IL-10 depresses iNOS and thus downregulates NO production, resulting in disease promotion (Huang et al., 1998). Raida and Buchmann, (2008) found that $Y$. ruckeri $\mathrm{O} 1$ amount in the spleen was correlated to the expression of cytokines genes $1 \beta$, IL-8, and IL-10 in rainbow trout. Moreover, the infected fish showed a significant rise in the transcript levels of chemokines, central cytokines, and cytokine receptors (IL- $1 \beta$, IL-6, IL-8, IL-10, TNF- $\alpha$, IL-receptor II) (Raida et al., 2011).

\section{CONCLUSION}

Thus it could be concluded that the pathogenesis of Yersiniosis in the cultured Oreochromis niloticus has been investigated in terms of clinical signs, pathogenicity patterns, and disturbances of hematological, and immunological responses. Also, Ascorbic Acid supplementation and Difloxacine dosing have proven their efficiency against Yersinia ruckeri infection indicated in improving health status and enhancement of biochemical and immune responses. Therefore ascorbic acid should be included within the preventive programs.

\section{REFERENCES}

Abd-EI Latief, J. I. (2001). Yersinia microorganisms as the causative agent of enteric red mouth disease in delta Nile fishes. MVSc thesis, Faculty of Veterinary Medicine, Cairo University. 
Abdel-Aziz, M.; Bessat, M.; Fadel, A. and Elblehi, S. (2020). Responses of dietary supplementation of probiotic effective microorganisms (EMs) in Oreochromis niloticus on growth, hematological, intestinal histopathological, and antiparasitic activities. Aquaculture International, pp.1-17.

Adams, C.; Braithwaite, V.; Huntingford, F.; Kadri, S.; Pottinger, T. and Turnbull, J. (2002). Fish Welfare . Briefi ng Paper 2, Fisheries Society of the British Isles, High Street, Sawston, Cambridge, 21 pp.

Afonso, A.; Lousada, S.; Silva, J.; Ellis, A. E. and Silva, M. T. (1998). Neutrophil and macrophage responses to inflammation in the peritoneal cavity of rainbow trout Oncorhynchus mykiss. A light and electron microscopic cytochemical study. Dis Aquat Organ, 34(1):27-37.

Altun, S. and Diler, O. (1999). Some hematological parameters of the rainbow trouts experimentally infected with Yersinia ruckeri.Turk. J. Vet. Anim. Sci., 23:301-309.

Aly, S. M. (2009). Probiotics and aquaculture. CAB Review: Perspectives in Agriculture, Veterinary Science, Nutrition and Natural Resources, 403: 1-16.

Anderson, D. P.; Dixon, O.W. and Roberson, B. S. (1979). Kinetics of the primary immune response in rainbow trout after flush exposure to Yersinia ruckeri Oantigen. Dev Comp Immunol 3:739-744.

Anderson, D. P.; Moritomo, T. and de Grooth, R. (1992). Neutrophil, glass-adherent, nitroblue tetrazolium assay gives early indication of immunization effectiveness in rainbow trout. Veterinary Immunology and Immunopathology 30(4):419-429.

Ayub, S.; Verma, J. and Das, N. (2003). Effect of endosulfan and malathion on lipid peroxidation, nitrite and TNF-alpha release by rat peritoneal macrophages. Int Immunopharmacol 3(13-14):1819-1828.

Barman, D.; Nen, P.; Mandal, S. and Kumar, V. (2013). Immunostimulants for Aquaculture Health Management.

Barrows, F. T.; Gaylord, T. G.; Sealey, W. M.; Porter, L. and Smith, C. E. (2008). The effect of vitamin premix in extruded plant-based and fish meal based diets on growth efficiency and health of rainbow trout, Oncorhynchus mykiss. Aquaculture, 283(1-4): 148155.

Bartels, H. and Bohmer, M. (1972). Clinical Chemistry. Acta 37:193.

Bartholomew, R. J. and Delaney, A. M. (1964). Spectrophotometric studies and analytical applications of the protein error of some $\mathrm{pH}$ indicators. Proc. Aust. Assoc. Clin. Biochem., $1: 64$.

Bastardo, A.; Sierralta, V.; León, J.; Ravelo, C. and Romalde, J. L. (2011). Phenotypical and genetic characterization of Yersinia ruckeri strains isolated from recent outbreaks in farmed rainbow trout Oncorhynchus mykiss (Walbaum) in Peru. Aquaculture, 317(1-4): 229-232.

Blaxhall, P. C. and Daisley, K. W. (1973). Routine hematological methods for use with fish Blazer, V. S. (1992). Nutrition and disease resistance in fish. Annual Review of Fish Diseases, 2, 309-323. blood. J. Fish Biol., 5: 771-781. 
Bullock, G. L. and Cipriano, R. C. (1990). Fish Disease Leaflet 82. Enteric Redmouth Disease of Salmonids. Fish and Wildlife Service Kearneysville WV National Fish Health Research Lab.

Bullock, G. L.; Struckey, H. M. and Shotts,Jr. E. B. (1977). Early records of North American and Australian outbreaks of enteric redmouth disease. Fish Health News, 6(2):96.

Cabello, F. C. (2006). Heavy use of prophylactic antibiotics in aquaculture: a growing problem for human and animal health and for the environment. Environmental microbiology, 8(7): 1137-1144.

Campos-Perez, J. J.; Ellis A. E. and Secombes C. J. (2000). Toxicity of nitric oxide and peroxynitrite to bacterial pathogens of fish. Dis Aquat Organ 43:109-115.

Clinical and Laboratory Standards Institute (CLSI), (2005a). Performance Standards For Antimicrobial Susceptibility Testing: Fifteen Informational Supplement CLSI, document M100-S15 (ISBN 1-56238-556-9). Clinical and Laboratory Standards Institute, Wayne, Pennsylvania 19087-1898, USA.

Clinical and Laboratory Standards Institute (CLSI), (2005b). Methods for antimicrobial disk susceptibility testing of bacteria isolated from aquatic animals; Proposed guidline. CLSI document M42-P (ISBN 1-56238-576-3). Clinical and Laboratory Standards Institute, Wayne, Pennsylvania. 41 pp, USA.

Cossarini-Dunier, M. (1986). Secondary response of rainbow trout to DNP haemocyanin and $Y$. ruckeri. Aquaculture 52:81-86.

Daligault, H. E.; Davenport, K. W.; Minogue, T. D.; Bishop-Lilly K. A.; Broomall, S. M.; Bruce, D. C.; Chain, P. S.; Coyne, S. R.; Frey, K. G.; Gibbons, H. S.; Jaissle, J.; Koroleva, G. I.; Ladner, J. T.; Lo, C. C.; Munk, C.; Palacios, G. F.; Redden, C. L.; Rosenzweig, C. N.; Scholz, M. B. and Johnson, S. L. (2014). Whole-genome Yersinia sp. assemblies from 10 diverse strains. Genome Announc., 2(5).

Danley, M. L.; Goodwin, A. E.; and Killian, H. S. (1999). Epizootics in farm-raised channel catfish, Ictalurus punctatus (Rafinesque), caused by the enteric redmouth bacterium Yersinia ruckeri. Journal of Fish Diseases, 22(6): 451-456.

Dorafshan, S.; Kalbassi, M. R.; Pourkazemi, M.; Mojazi-Amiri, B. and Soltan-Karimi, S. (2008). Effects of triploidy on the Caspian salmon (Salmo trutta, caspius) haematology. Fish Physiol. Biochem., 34: 195-200.

Duncan, B. (1955). Multiple Range and Multiple (F) tests. Biometrics, 11: 1- 2.

Ellis, A. E. (1981). Non-specific defense mechanisms in fish and their role in disease processes. . Dev Biol Stand., 49:337-352.

Ellis, A. E.; in: Stolen, J. S.; Fletcher, T. C.; Anderson, D. P.; Robertson, B. S. and Van, W. R. (1990). Muisvinkel (Eds.), Lysozyme assay in techniques in fish immunology, SOS publications, Fair Haven, NJ, USA.:pp.101-103.

Elmeer, W. K.; Stephen, D. A.; William, M. J.; Paul, C. S. and Washington, C. W. (1997). Color Atlas and Textbook of Diagnostic Microbiology. $5^{\text {th }}$ Ed. Lippincott. Philadelphia. New York.

Elston, R. A.; Drum, A. S.; Pearson, W. H. and Parker, K. (1997). Health and condition of pacific herring Clupea pallasi from Prince William Sound, Alaska, from 1994. Diseases of aquatic organisms, 31(2): 109-126.

Ergönül, M. B.; Yavuzcan, H. and Altındağ, A. (2012). Balık sağlığı ve immunostimülantların kullanımı. Journal of Fisheries Sciences, 6 (3): 188-202. 
Fernandez-Dıaz, M. D.; Montero, P. and Gómez-Guillén, M. C. (2003). Effect of freezing fish skins on molecular and rheological properties of extracted gelatin. Food hydrocolloids, 17(3), 281-286.

Fuchs, R.; Appelgren, L. E. and Hult, K. (1986). Distribution of 14C-ochratoxin A in the rainbow trout (Salmo gairdneri). Acta Pharmacol Toxicol, 59:220-227.

Fuhrmann, H., Bohm, K. H. and Schlotfeldt, H. J. (1983). An outbreak of enteric redmouth disease in West Germany. J. fish Dis. 6: 30S311.

Furones, M. D.; Rodgers, C. J. and Munn, C. B. (1993). Yersinia ruckeri, the causal agent of enteric redmouth disease (ERM) in fish. Annual Review of Fish Diseases, 3, 105-125.

Glasser, L. and Fiederlein, R. L. (1990). The effect of various cell separation procedures on assays of neutrophil function. American Journal of Clinical Pathology, 93 (5): 662669.

Green, L. C.; Wagner, D. A.; Glogowski, J.; Skipper, P. L.; Wishnok, J. S. and Tannenbaum, S. R. (1982). Analysis of nitrate, nitrite, and 15-nitrate in biological fluids. Anal Biochem; 126: 131-8.

Guijarro, J. A.; García-Torrico, A. I.; Cascales, D. and Méndez, J. (2018). The Infection Process of Yersinia ruckeri: Reviewing the Pieces of the Jigsaw Puzzle. Frontiers in cellular and infection microbiology, 8, 218pp.

Hoffman, R.; Benz E. J.; Furie, B. and Shattil, S. J. (2009). Hematology: basic principles and practice. Churchill Livingstone, Philadelphia.

Horne, M. T. and Barnes, A. C. (1999). Enteric redmouth disease (Yersinia ruckeri). In: Woo PTK, Bruno DW (eds) Fish diseases and disorders. Viral, bacterial and fungal infections. CABI Publishing, Wallingford, pp 445-477.

Huang, F. P.; Xu, D.; Esfandiari, E. O. et al. (1998). Mice defective in Fas are highly susceptible to Leishmania major infection despite elevated IL-12 synthesis, strong Th1 responses, and enhanced nitric oxide production. J Immunol; 160: 4143-7.

Hunter, V. A.; Knittel, M. D. and Fryer, J.L. (1980). Stress-induced transmission of Yersinia ruckeri infection from carriers to recipient steelhead trout Salmogairdneri richardson. J Fish Dis, 3:467-472.

Hussein, M. M.; Elkhatibe, N. R. and Riad, E.M. (1997). Studies on enteric redmouth disease among freshwater fish. Veterinary Medical Journal, 45, 549-559.

Jaafar, R. M.; Kania, P.; Larsen, A. H.; Nielsen, D.; Fouz, B.; Browdy, C. and Buchmann, K. (2013). Gut microbiota changes in rainbow trout, Oncorhynchus mykiss (Walbaum), during organic acid feed supplementation and Yersinia ruckeri infection. Journal of Fish Diseases, 36 (6): 599-606.

Jollès, P. and Jollès, J. (1984). What's new in lysozyme research? Molecular and Cellular Biochemistry, 63(2):165-189.

Juedes, M. J. and Wogan G.N. (1996). Peroxynitrite-induced mutation spectra of pSP189 following replication in bacteria and in human cells, Mutat. Res. 349: 51-61.

Klein, B. U.; Siesenop, U. and Boehm, K. H. (1996). Investigations on transferable antibiotic resistance through R-plasmids between obligate and facultative fish pathogenic bacteria. Bull. Eur. Assoc. Fish Pathol. 16:138-142.

Koller, A. (1984). Total serum protein . in Kaplan L.A. , Pesce A.J. (ed.). Clinical Chemistry, Theory, Analysis , and Correlation. St. Louis: Mosby Company , pp:1316-1319 . 
Kumar, G.; Menanteau-Ledouble, S.; Saleh, M. and El-Matbouli, M. (2015). Yersinia ruckeri, the causative agent of enteric redmouth disease in fish. Vet Research. 46:103.

Kumari, J. and Sahoo, P. K. (2006). Dietary b-1,3 glucan potentiates innate immunity and disease resistance of Asian cat fish, Clarias batrachus (L.). Journal of Fish Diseases 29, 95-101.

Lall, S. P. (2000). Nutrition and health of fish. Avances en nutrición acuícola.19-22.

Mammeri, H.; Poirel, L.; Nazik, H. and Nordmann, P. (2006). Cloning and functional characterization of the ambler class $\mathrm{C}$ beta-lactamase of Yersinia ruckeri. FEMS Microbiol Lett, 257:57-62.

Méndez, J. and Guijarro, J. A. (2013). In vivo monitoring of Yersinia ruckeri in fish tissues: progression and virulence gene expression. Environmental Microbiology Reports, 5(1), 179-185.

Michel, C.; Nougayrede, P.; Eldar, A.; Sochon, E. and De Kinkelin, P. (1997). Vagococcus salmoninarum, a bacterium of pathological significance in rainbow trout Oncorhynchus mykiss farming. Diseases of Aquatic Organisms, 30(3):199-208.

Murray, C. K. and Fletcher, T. C. (1976). The immunohistochemical localization of lysozyme in plaice (Pleuronectes platessa L.) tissues. J Fish Biol, 9: 329-334.

Nathan, C.F. (1987). Neutrophil activation on biological surfaces. Massive secretion of hydrogen peroxide in response to products of macrophages and lymphocytes. J. Clin. Invest. 80, 1550-1560.

Navas, E.; Bohle, H.; Henríquez, P.; Grothusen, H.; Bustamante, F.; Bustos, P. and Mancilla, M. (2014). Draft genome sequence of the fish pathogen Yersinia ruckeri strain 37551, serotype O1b, isolated from diseased, vaccinated Atlantic salmon (Salmo salar) in Chile. Genome Announc 2:e00858-14.

Ohtani, M.; Villumsen, K. R.; Strøm, H. K. and Raida, M. K. (2014). 3D visualization of the initial Yersinia ruckeri infection route in rainbow trout (Oncorhynchus mykiss) by optical projection tomography. PLoS One, 9(2), e89672.

Pham, C. K.; Vandeperre, F.; Menezes, G.; Porteiro, F.; Isidro, E. and Morato, T. (2015). The importance of deep-sea vulnerable marine ecosystems for demersal fish in the Azores. Deep Sea Research Part I: Oceanographic Research Papers, 96, 80-88.

Puangkaew, J.; Kiron, V.; Somamoto, T.; Okamoto, N.; Satoh, S.; Takeuchi, T. and Watanabe, T. (2004). Nonspecific immune response of rainbow trout (Oncorhynchus mykiss Walbaum) in relation to different status of vitamin E and highly unsaturated fatty acids. Fish Shellfish Immunol. 16:25-39.

Ra,a J.T. (1994). Abnormalities of plasma enzymes. Scientific Foundations of Clinical Biochemistry Biochemistry in Clinical Practice, Williams DL, Marks V (Eds), $2^{\text {nd }}$ Edition, William Heinemann, London 2:221-250.

Rahimi Yadkoori, N.; Zanguee, N.; Mousavi, S. M. and Zakeri, M. (2015). Effects of ginger (Zingiber officinale) extract on digestive enzymes and liver activity of Mesopotamichthys sharpeyi fingerlings. Journal of the Persian Gulf (Marine Science), 6(19), 1-10

Raida, M. K. and Buchmann K. (2008). Development of adaptive immunity in rainbow trout, Oncorhynchus mykiss (Walbaum) surviving an infection with Yersinia ruckeri. Fish Shellfish Immunol. 25: 533-541.

Raida, M. K.; Holten-Andersen, L. and Buchmann, K. (2011). Association between Yersinia ruckeri infection, cytokine expression and survival in rainbow trout (Oncorhynchus mykiss). Fish Shellfish Immunol. 30: 1257-1264. 
Reitman, S. and Frankel, S. (1957). Transaminase in serum. Amer. J. Clin. Path. 25-56.

Rigos, G. and Stevenson, R. (2001). The effect of antibiotic treatment on the establishment of persistent infection with Yersinia ruckeri Serovar II in rainbow trout Oncorhynchus mykiss (Walbaum). Aquacult. Int., 9: 247- 253.

Rodgers, C. J. (2001). Resistance of Yersinia ruckeri to antimicrobial agents in vitro. Aquaculture 196:325-345.

Roed, K. H.; Larsen, H. J.; Linder, R. D. and Refstie, T. (1993). Genetic variation in lysozyme activity in rainbow trout (Oncorhynchus mykiss). Aquaculture, 109:237-244.

Romalde, J. L.; Barja, J. L.; Magarinos, B. and Toranzo, A. E. (1994). Starvation-survival processes of the bacterial fish pathogen Yersinia ruckeri. Syst. Appl. Microbiol. 17:161168.

Ross, A. J.; Rucker, R. R. and Ewing, W.H. (1966). Description of a bacterium associated with red mouth disease of rainbow trout (Salmo gairdneri). Can J Microbiol, 12:763-770.

Salah, M.; Wael G. and Mounir M. (2012). Bacteriological and Histopathological Studies on Enterobacteriacea in Nile Tilapia Oreochromis Niloticus. J. Pharm. Biomed. Sci., 2(7):94104.

SAS, (2005). Statistical Analysis System. User`s Guide: SAS Institute Cary, North Carolina.

Saurabh, S. and Sahoo, P. K. (2008). Lysozyme: an important defence molecule of fish innate immune system. Aquaculture Research, 39(3):223-239.

Shaowu L.; Di, W.; Hongbai, L. and Tongyan, L. (2013). Isolation of Yersinia ruckeri strain H01 from farm-raised amur sturgeon Acipenser schrencki in China. J Aquat Anim Health, 25:9-14.

Shiau, Shi-Yen, and Chun-Yi Hsu (2002). Vitamin E sparing effect by dietary vitamin C in juvenile hybrid tilapia, Oreochromis niloticus "O. aureus." Aquaculture, 210, 1-4: 335342.

Smith, M. L. (1988). Effects of observer swimming speed on sample counts of temperate rocky reef fish assemblages. Marine ecology progress series. Oldendorf, 43(3), 223-231.

Sogo, T.; Kawahara, M.; Ueda, H.; Otsu, M.; Onodera, M.; Nakauchi, H. et al. (2009). T cell growth control using hapten-specific antibody/interleukin-2 receptor chimera. Cytokine; 46(1):127-36.

Stock, I.; Henrichfreise, B. and Wiedemann B. (2002). Natural antibiotic susceptibility and biochemical profiles of Yersinia enterocolitica-like strains: Y. bercovieri, $Y$. mollaretii, $Y$. aldovae and "Y. ruckeri.". J Med Microbiol, 51:56-69.

Suresh, V. (2003). Tilapias. In: Aquaculture: farming aquatic animals and plants [ed. by Lucas, J. S. ISouthgate, P. C.]. Oxford, UK: Blackwell Publishing.

Tewary, A. and Patra, B.C. (2008). Use of vitamin C as an immunostimulant effect on growth, nutritional quality, and immune response of Labeo rohita (Ham). Fish Physiology and Biochemistry, 34(3): 251-9.

Tietz, N. W. (1995). Clinicl Guide to Laboratory Tests. $3^{\text {rd }}$ Ed. Philadelphia, WB Saunders Company.pp: 622-629.

Tobback, E.; Decostere, A.; Hermans, K.; Haesebrouck, F. and Chiers K. (2007). Yersinia ruckeri infections in salmonid fish. J Fish Dis 30:257-268. 
Tobback, E.; Decostere, A.; Hermans, K.; Ryckaert, J.; Duchateau, L.; Haesebrouck, F. and Chiers, K. (2009). Route of entry and tissue distribution of Yersinia ruckeri in experimentally infected rainbow trout Oncorhynchus mykiss. Dis Aquat Org,84:219-228.

Van Doan, H.; Hoseinifar, S. H.; Tapingkae, W.; Seel-Audom, M.; Jaturasitha, S.; Dawood, M. A. and Esteban, M. Á. (2020). Boosted growth performance, mucosal and serum immunity, and disease resistance Nile tilapia (Oreochromis niloticus) fingerlings using corncob-derived xylooligosaccharide and Lactobacillus plantarum CR1T5. Probiotics and antimicrobial proteins, 12(2), 400-411.

Waltman, W. D.; and Shotts Jr, E. B. (1984). A medium for the isolation and differentiation of Yersinia ruckeri. Canadian Journal of Fisheries and Aquatic Sciences, 41(5), 804-806.

Wang, T.; Bird, S.; Koussounadis, A.; Holland, J. W.; Carrington, A.; Zou, J. and Secombes, C. J. (2009). Identification of a novel IL-1 cytokine family member in teleost fish. J Immunol, 183: 962- 974.

Xia, Y.; Wang, M.; Gao, F.; Lu, M. and Chen, G. (2020). Effects of dietary probiotic supplementation on the growth, gut health and disease resistance of juvenile Nile tilapia (Oreochromis niloticus). Animal Nutrition, 6(1): 69-79.

Young, D. S. (2001). Effect of Disease on Clinical Lab. Test. $4^{\text {th }}$ ed, AACC.

Zhang, Z., Swain T.; Bogwald J.; Dalmo R.A. and Kumari, J. (2009). Bath immunostimulation of rainbow trout (Oncorhynchus mykiss) fry induces enhancement of inflammatory cytokine transcripts, while repeated bath induce no changes. Fish Shellfish Immunol.; 26(5):677-8. 